\title{
Bio Inspired Modified Internal Model Control Approach for Improved Disturbance Rejection of Piezo Micro Manipulator
}

\author{
Saikat Kumar SHOME ${ }^{1,2 *}$, Sandip JANA², Arpita MUKHERJEE ${ }^{1,2}$, \\ Partha BHATTARCHARJEE ${ }^{2}$, Uma DATTA ${ }^{1,2}$ \\ ${ }^{1}$ Academy of Scientific and Innovative Research (AcSIR), CSIR- Central Mechanical Engineering \\ Research Institute (CSIR-CMERI) Campus, Durgapur 713209, India \\ ${ }^{2}$ CSIR-Central Mechanical Engineering Research Institute, Ministry of Science and Technology, \\ Govt of India, Durgapur 713209, India \\ saikatkumarshome@gmail.com (*Corresponding author)
}

\begin{abstract}
Classical Internal Model Control based approach for controller design has been used in different industrial control applications as it allows good set point tracking performance, especially for processes neglecting time delay. However, in many process control applications including nonlinear piezo electric actuation (PZA), disturbance rejection plays an important role compared to set point tracking. The present research firstly proposes an optimal filter design in series with a Modified Internal Model Control (M-IMC) based Proportional -Integral-Derivative (PID) controller for better set point tracking, improved disturbance rejection with reduced controller hardware resource requirement compared to classical IMC. Two efficient swarm intelligence based evolutionary soft computational techniques viz. Particle Swarm Optimization (PSO) and Bacterial Foraging Optimization (BFO) are then exploited towards optimizing a control evaluation index based fitness function to design the M-IMC control parameters, including filter time constant. The distillation of bio inspired principles in control is seen to exhibit exciting results when the optimized parameters are utilized in the piezo plant modeled using a Dahl based second order system. The performance of the controller has been evaluated by subjecting the plant to several perturbations as well as to external disturbances. The results illustrate the efficiency of the PSO based M-IMC over other controllers.
\end{abstract}

Keywords: Piezo actuator, Internal Model Control, Swarm, Disturbance, Dahl Model, PSO, BFO

\section{Introduction}

Accurate positioning applications are pervasive in industrial and manufacturing applications, arising with prolific growth of nanoscale applied science. In the recent past, piezoelectric actuators (PZAs) have proved themselves to be major players in the realm of precision positioning $[25,30]$. They have emanated as one of the most widely used type of actuators in this area due to advantages such as increased electromechanical coupling efficiency, enhanced resolution, quick frequency domain response, large stiffness coefficient, and minimal thermal expansion in the process of actuation $[6,2]$. Industrially interactive scientific micro/ nano manipulation applications generally call for control strategies which are not only capable of providing precise reference tracking but are also robust to external force [3, 28]. Simulation and modelling of such high accuracy industrial servo mechanisms that predicts and optimizes both static and dynamic performances of the systems under study are seen in [23, 24].

These essentialities are further effected by the inherent nonlinearities like hysteresis which hinder precision positioning capabilities.
Thus, it is necessary to design decisive control methodologies for PZAs in order to satiate the constantly rising design requirements. Extensive research has been carried out with regard to implementation of appropriate control structures to take into consideration piezoelectric hysteresis and other nonlinearities. PI and PID have been seen to be the most popular classical controllers however with limited bandwidth and, therefore useful for macro scale applications without predominant disturbances.

Of late, model based control regimes like the dynamic matrix control; adaptive reference control and predictive control are achieving acceptance as presented in [12]. In this case, the feedback controller is generally designed by employing a model of the physical process which is to be controlled along with the dynamics of the external perturbations affecting the system flow. A model-based control algorithm named "Internal Model Control" (IMC) which is centred around the principle of internal mathematical model of the process and its inverse dynamics have gained considerable popularity $[9,10]$. 
There have been researches reported on IMC pertaining to several independent industry oriented applications, based on the analysis of the control system's stability and robust behaviour $[9,18]$ without any study dedicated towards utilising the efficacy of IMC for PZA based applications. An impediment of traditional IMC design is the replication of the plant models in hardware which increases the hardware requirement. Besides, in the event of a mismatch between the plant model and actual process, there is a degradation in closed-loop performance which also needs to be compensated along with proper tuning of the filter time constant.

Bio-inspired optimization algorithms using soft computing and artificial intelligence play a significant role in optimization of parameters [14]. The primary motivation utilizing AI technology is to make the system behave in a more dynamic manner similar to human mind. Efficiency, performance and cost have become ubiquitous in the modern industrial era with most applications demanding a minimizing cost or maximizing performance optimization problem. In a wide class of problems, there is a requirement to deduce an optimal solution owing to more than one characteristic. In such cases, multi-objective approach needs to be used wherein the several objectives are either accommodated in a single fitness function or each of them captured through distinct tasks $[8,13]$.

The proposed research is based on exploiting Particle Swarm Optimization (PSO) and Bacterial Foraging Optimization (BFO), two well accepted and efficient swarm intelligence- based evolutionary soft computational techniques which employ a multi-objective approach. They are used to optimise the filter time constant and the gain values to PID equivalent Modified Internal Model Controller, through a fitness function. The prudence behind selecting these two attractive algorithms is that they do not get largely impaired by the population size or the non-linear nature of the problem with a better convergence rate that most analytical methods [21]. These algorithms also have the advantages of being computationally inexpensive using basic mathematical operators, global convergence and low memory requirement compared to the other evolutionary algorithms.
The rest of the paper is organised as follows: Section 2 briefs about the theoretical background of Internal Model control and the design of their fitness function. Section 3 presents an overview of BFO and PSO along with its application on PZA system. The results and discussion on the performance of the controllers are detailed in Section 4 which is followed by the concluding remarks.

\section{Internal Model Control}

\subsection{Classical Internal Model Control}

Internal model controller relates to a modelbased process where the mathematical model of the process is embedded inside the controller in a closed loop. The IMC philosophy constitutes the process model, an inverse model and a filter as reflected in Figure 1 where $G_{\text {Piezo }}(s)$ stands for the process, $\tilde{G}_{\text {Piezo }}(s)$ represents the mathematical model of the process while $G_{I n v-\text { Piezo }}(s)$ corresponds to the transfer function of the IMC controller and $d(s)$ is the external perturbations affecting the system [12].

The aim of the parallel loop containing the plant model is to obtain a difference between the real plant and its estimated transfer function leading to

$\hat{d}(s)=\left[G_{\text {Piezo }}(s)-\tilde{G}_{\text {Piezo }}(s)\right] U(s)+d(s)$

The error signal facilitates in achieving the desired set-point taking into consideration the system model mismatch and external disturbances. The resulting control signal is thus given by:

$$
\begin{aligned}
& U(s)=[\mathrm{R}(\mathrm{s})-\hat{d}(s)] G_{\text {Inv-Piezo }}(s) \\
& =\left\{R(\mathrm{~s})-\left[\mathrm{G}_{\text {Piezo }}(s)-\widetilde{G}_{\text {Piezo }}(s)\right] U(s)+d(s)\right\} G_{\text {Inv-Piezo }}(s) \\
& U(s)=\frac{[R(s)-d(s)] G_{\text {Inv }- \text { Piezo }}(s)}{\left[1+G_{\text {Piezo }}(s)-\widetilde{G}_{\text {Piezo }}(s)\right] G_{\text {Inv-Piezo }}(s)}
\end{aligned}
$$

Also, $Y(s)=G_{\text {Piezo }}(s) U(s)+d(s)$

Closed loop transfer function of IMC is obtained by substituting into $\mathrm{Y}(\mathrm{s})$

$$
Y(s)=\frac{[R(s)-d(s)] G_{\text {Inv-Piezo }}(s) \mathrm{G}_{\text {Piezo }}(s)}{\left[1+G_{\text {Piezo }}(s)-\widetilde{G}_{\text {Piezo }}(s)\right] G_{\text {Inv-Piezo }}(s)}+d(s)
$$

Thus, it can be derived that a) if $G_{I I v-\text { Piezo }}(s)=\tilde{G}_{\text {Piezo }}(s)$ and $\tilde{G}_{\text {Piezo }}(s)=G_{\text {Piezo }}(s)$, then absolute set point tracking along with disturbance 
rejection is achievable, b) if $G_{\text {Piezo }}(s) \neq \tilde{G}_{\text {Piezo }}^{-1}(s)$, disturbance rejection can still be achieved if $G_{\text {Inv-Piezo }}(s)=\tilde{G}_{\text {Piezo }}^{-1}(s)$.

An advantage of IMC is that it involves a single tune able parameter which is the filter time constant. Variation in this constant abruptly affects the system control performance. This study attempts to design this important coefficient using swarm-based intelligent algorithms.

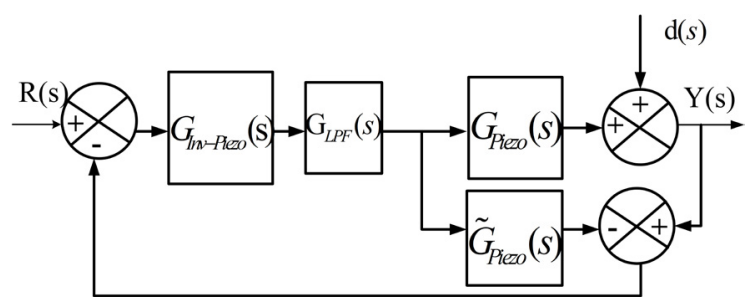

Figure 1. Classical IMC structure for piezo electric actuator system

\subsection{Modified Internal Model Control (M-IMC) for Piezo Electric Actuation System}

The transparent framework presented in the previous section based on classical Internal Model Control has a limitation. The structure was realized by first placing the PZA plant in the loop, followed by reusing it as a reference model in a parallel. This involves replicative work first for the model and second for the controller, thus engaging significant controller hardware. Modified Internal Model Control proposes few alterations in the generic IMC structure to minimize control hardware requirement. Although IMC can be easily implemented, yet Proportional Integral Derivative control remains the most commonly engaged industrial controller. Hence, a PID equivalent structure of M-IMC is presented. The transformation to Modified IMC has been diagrammatically illustrated in Figure 2. The IMC based process can hence be utilized in the design of a standard feedback controller based upon the internal PZA model and Internal Model Controller, $G_{\text {Inv-Piezo }}(s)$. $\mathrm{R}(\mathrm{s})-\mathrm{Y}(\mathrm{s})$ is the generalized error term used by a typical feedback control structure. Accordingly, this reformulation is also advantageous as it offers a lighter IMC framework along with a PID equivalence which finds usage in common industrial applications.

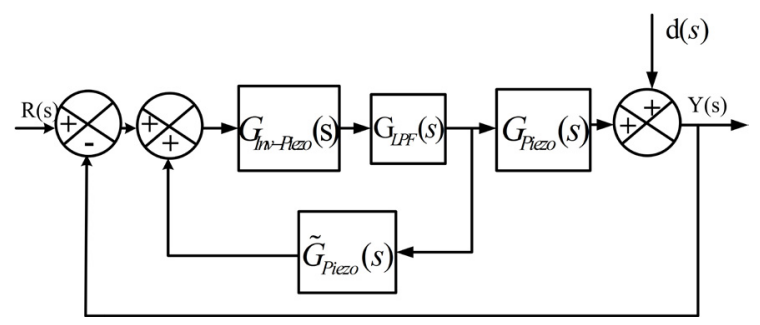

Figure 2. Modified IMC structure for piezo electric actuator system.

\subsubsection{The Filter Transfer Function}

In the PZA system, a low pass filter is augmented with the improper IMC controller $G_{\text {Inv-Piezo }}(s)$ to make it proper and also to enhance the system robustness because the gap between the process and its model is pronounced at the higher end of the frequency response. This robust filter is crucial in the controller design of the PZA system by considering several control performance issues in the final design so that the targeted controller can obtain the required design specifications - robustness in performance and stability. Introduction of the filter also makes the controller stable, causal and proper. The final shape of the controller inclusive of the augmented filter function is as

$$
G_{\text {Inv-Piezo }}(s)=\tilde{G}_{\text {Piezo- }^{-1}}^{-1}(s) \cdot \mathrm{G}_{L P F}(s) \text {,with }
$$

$G_{L P F}(s)=\frac{1}{(\alpha s+1)^{n}}$ where ' $\mathrm{n}$ ' is the filter order and $\alpha$ is the filter time-constant.

\subsubsection{PID Equivalent form of Modified- IMC for PZA System}

Let the PZA system be represented by a 2 nd order process as follows:

$\tilde{G}_{\text {Piezo }}(\mathrm{s})=\frac{k}{\left(\tau_{1} s+1\right)\left(\tau_{2} s+1\right)}$

The PZA plant is factorized into minimum and non-minimum phase elements. The later are considered for the inverse controller, which is then augmented with a low pass filter as seen in the following equation

$$
\begin{aligned}
& G_{I n v-\text { Piezo }}(s)=\tilde{G}_{I n v-\text { Piezo }}(s) G_{L P F}(s) \\
& =\tilde{G}_{P i e z o}^{-1}(s) G_{L P F}(s)=\frac{\left(\tau_{1} s+1\right)\left(\tau_{2} s+1\right)}{k(\alpha s+1)}
\end{aligned}
$$

where $G_{L P F}(s)$ is the low pass filter. 
The equation of the equivalent standard closed loop controller is obtained using the transformation

$$
\begin{aligned}
& G_{M-I M C}(s)=\frac{G_{\text {Inv-Piezo }}(s)}{1-\tilde{G}_{\text {Piezo }}(s) G_{\text {Inv-Piezo }}(s)} \\
& =\frac{\frac{\left(\tau_{1} s+1\right)\left(\tau_{2} s+1\right)}{k(\alpha s+1)}}{1-\frac{\left(\tau_{1} s+1\right)\left(\tau_{2} s+1\right)}{k(\alpha s+1)}} \\
& =\frac{\tau_{1} \tau_{2} s^{2}+\left(\tau_{1}+\tau_{2}\right) s+1}{k \alpha s}=\frac{\tau_{1} \tau_{2}}{k \alpha} s+\frac{\left(\tau_{1}+\tau_{2}\right)}{k \alpha}+\frac{1}{k \alpha s}
\end{aligned}
$$

Transfer function of a classical PID controller is

$$
g_{c}(s)=\left(K_{P}+\frac{K_{I}}{s}+K_{D} s\right)
$$

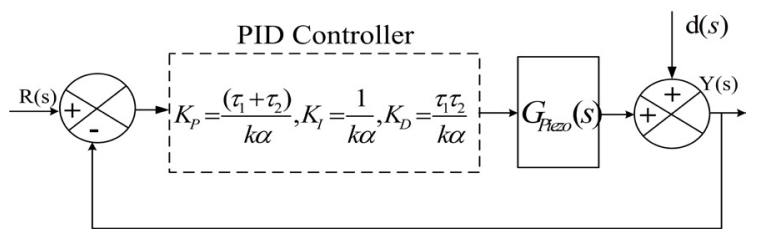

Figure 3. PID Equivalent Control of Modified IMC for PZA.

Equating the above equations leads us to the PID equivalent form of the M-IMC, represented in Figure 3.

\subsection{Determination of the PZA Design Fitness Function}

IMC offers an important advantage in choosing a single tunable design parameter in the form of filter coefficient. In this investigation, the Modified IMC's PID equivalent controller is tuned using bio inspired swarm intelligence techniques. The soft computing methodologies have been mainly used to regulate the optimal filter constant which in turn has been used to tune the PID parameters. A three dimensional search space is defined in which each dimension stands for each of the PID parameters. A definite point in the search space corresponds to a particular combination of $\left[\mathrm{K}_{\mathrm{P}} \mathrm{K}_{\mathrm{I}} \mathrm{K}_{\mathrm{D}}\right]$ for which a distinct output response is obtained. The performance of the point is determined by a fitness function comprising several components which serve as control evaluation index of the design. The point in the search space is considered the global best if the fitness function attains an extreme value for it i.e. either a maxima or a minima.

In this design, four components have been used to define the fitness function - settling time, peak overshoot, steady state error and rise time. The contribution of each of these four constituents towards the original fitness function is decided by a scale factor whose choice is decided by the designer. As each of these functions are individually minimized in nature, the best design point for the optimal response characteristics corresponds to the minimal value of the overall fitness function.

As elaborated, mathematically the fitness function is represented as:

$F=\left[1-e^{(-\eta)}\right]\left(M_{P}+E_{S S}\right)+\left[e^{(-\eta)}\right]\left(T_{S}-T_{R}\right)$

Where F- Fitness function, $\mathrm{M}_{\mathrm{P}}$ - Peak Overshoot, $\mathrm{T}_{\mathrm{S}}$ - Settling Time, $\mathrm{T}_{\mathrm{R}}$ - Rise Time, $\eta$ :-Scaling Factor, and the scaling factor as $\eta$.

The point in the search space is considered as the best point if the fitness function attains a minimum value. The value of this fitness function has been used as different combination of the gains correspondingly reflected in three dimensional search space, and optimized through two swarm intelligence techniques - Bacterial Foraging Optimization and Particle Swarm Optimization, as seen in Figure 4.

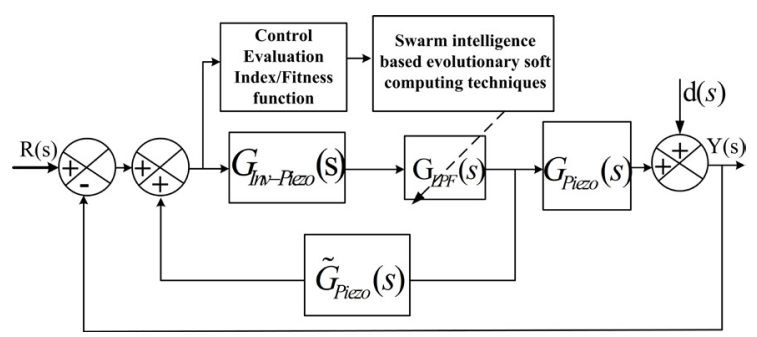

Figure 4. Block Schematic of Swarm Intelligence Optimized Modified Internal Model Control for PZA system.

\section{Swarm Intelligence-Based Soft Computational Techniques for Filter Time Constant Optimization}

\subsection{Bacterial Foraging Optimization Algorithm (BFO)}

Based on the study of foraging behavior of E.coli bacteria, a relatively new algorithm named Bacterial Foraging Optimization was introduced in the family of bio-nature inspired optimization techniques $[21,11]$. The work analyzed a wide gamut of bacterial foraging and swarming behavior 
and discussed how to relate distributed nongradient optimization with biological and social foraging process. The primary motive of this new philosophy is the exploitation of group foraging strategy of a bacterial swarm for multi-optimal function optimization $[5,17]$. To maximize the amount of energy obtained with respect to time, bacteria search for nutrients with even individual bacterium communicating with each other by sending signals. Taking into consideration the previous factors foraging decisions are taken by a single bacterium.

\subsubsection{Application of Bacterial Foraging Optimization Algorithm for M-IMC}

The BFO based simulation has been carried out using the following algorithm and parameters as presented in Table 1. The fitness function decreases as the number of bacteria in the population is increased, leading to an optimized value of 8.1652 for a population of size 1000 , as seen in Table 2.

The Algorithm

Step 1: Parameters $\mathrm{P}, \mathrm{S}, \mathrm{S}_{\mathrm{t}}, \mathrm{S}_{\mathrm{r}}, \mathrm{S}_{\mathrm{e}}, \mathrm{P}_{\mathrm{e}}$ and the $\mathrm{C}$ (i), $(\mathrm{i}=1,2, \ldots, \mathrm{P})$ are initialized. Initialize $\phi^{i}, \mathrm{i}=1,2 \ldots$ P. The control variables are randomly distributed throughout the search space. When $\phi$ is computed, the position of each bacterium in the population of the bacteria $\mathrm{P}$. is refreshed spontaneously along with a termination test for maximum number of stipulated loops.

Step 2: $\quad$ Elimination-Dispersal loop: $1=1+1$

Step 3: $\quad$ Reproduction loop: $\mathrm{k}=\mathrm{k}+1$

Step 4: $\quad$ Chemo taxis loop: $\mathrm{j}=\mathrm{j}+1$

(i) Chemotactic step for bacterium cell ' $i$ ' is taken, for $\mathrm{i}=1,2 \ldots, \mathrm{P}$ :

(ii) Cost $Z(i, j, k, l)$ is computed.

(ii) Assume

$Z(i, j, k, l)=Z(\mathrm{i}, \mathrm{j}, \mathrm{k}, 1)+Z_{c c}\left(\phi^{i}(j, \mathrm{k}, \mathrm{l}), \mathrm{P}(\mathrm{j}, \mathrm{k}, \mathrm{l})\right)$ to accumulate cell-to-cell attractant effect with nutrient concentration

(ii) Let $Z_{\text {last }}=Z(i, j, k, l)$ is used to store the present value as a better value may be resulted during the run (v) Tumble: A random vector is generated $\Delta(i) \in R^{p}$ with each element $\Delta_{m}(i), \mathrm{m}=1$, $2, \ldots, \mathrm{d}$ in the range $[-1,1], \mathrm{R}$ being a real number.

(vi) A step of size C(i) is simulated in the tumble direction for $i^{\text {th }}$ cell as

$\Phi^{i}(j+1, k, 1)=\Phi^{i}(j, k, 1)+C(\mathrm{i}) \frac{\Delta(i)}{\sqrt{\Delta^{T}(\mathrm{i}) \Delta(\mathrm{i})}}$

Table 1.BFO algorithm control parameters

\begin{tabular}{|l|l|l|}
\hline Sl.No & Parameters & Values \\
\hline 1 & $\begin{array}{l}\text { Number of bacterium in } \\
\text { population, P }\end{array}$ & $\begin{array}{c}100 \\
\text { (initial) }\end{array}$ \\
\hline 2 & $\begin{array}{l}\text { Maximum number of steps, } \\
\mathrm{S}_{\mathrm{t}}\end{array}$ & 3 \\
\hline 3 & $\begin{array}{l}\text { Number of chemo tactic } \\
\text { steps, } \mathrm{S}_{\mathrm{c}}\end{array}$ & 4 \\
\hline 4 & $\begin{array}{l}\text { Number of reproduction } \\
\text { steps, } \mathrm{S}_{\mathrm{r}}\end{array}$ & 6 \\
\hline 5 & $\begin{array}{l}\text { Number of elimination- } \\
\text { dispersal steps, } \mathrm{S}_{\mathrm{e}}\end{array}$ & 2 \\
\hline
\end{tabular}

Table 2.BFO based optimization result

\begin{tabular}{|c|c|}
\hline $\begin{array}{c}\text { Number of } \\
\text { bacterium in } \\
\text { population, } \mathbf{P}\end{array}$ & $\begin{array}{l}\text { Optimal Fitness } \\
\text { Function Value }\end{array}$ \\
\hline 100 & 16.2137 \\
\hline 200 & 14.6071 \\
\hline 300 & 14.0316 \\
\hline 400 & 13.1201 \\
\hline 500 & 12.8561 \\
\hline 600 & 11.4924 \\
\hline 700 & 10.5132 \\
\hline 800 & 9.6121 \\
\hline 900 & 8.4297 \\
\hline 1000 & 8.1652 \\
\hline
\end{tabular}

(vii) $Z(\mathrm{i}, \mathrm{j}+1, \mathrm{k}, \mathrm{l})$ is computed. In case of the loss or cost function being minimum, continue to next step else break to step (iii)

(viii) Swim - An approximation is made and swimming behavior ofeach cell is considered if the bacteria numbered $\{1,2, \ldots \ldots ., \mathrm{i}\}$ have moved and $\{i+1, i+2, i+3 \ldots . . . S\}$ have not

(a) Assume $m=0$ (swim length counter)

(b) While $\mathrm{m}<\mathrm{S}_{\mathrm{t}}$ 
Let $\mathrm{m}=\mathrm{m}+1$

If $Z(i, j+1, k, l)<Z_{\text {last }}$ (if there is betterment), let $Z_{\text {last }}=Z(\mathrm{i}, \mathrm{j}+1, \mathrm{k}, \mathrm{l})$

$\Phi^{i}(j+1, k, 1)=\Phi^{i}(j+1, k, 1)+C(\mathrm{i}) \frac{\Delta(i)}{\sqrt{\Delta^{T}(\mathrm{i}) \Delta(\mathrm{i})}}$

$\phi^{i}(j+1, k, l)$ is used to compute the new $Z(i, j+1, k, l)$

Else, let $\mathrm{m}=\mathrm{S}_{\mathrm{t}}$.

End of while loop.

(ix) Move to next cell (i+1) if $i \neq$ to $P$

Step 5: If $\mathrm{j}<\mathrm{S}_{\mathrm{c}}$, move to step 3. However, chemotaxis may be continued as the life of the bacteria still exists.

Step 6: Reproduction

a) For a particular $\mathrm{k}$ and 1 , and for every $\mathrm{i}=1,2,3,4 \ldots . . . \mathrm{P}$, let $Z_{\text {health }}^{i}=\sum_{j=1}^{S_{c}+1} Z(i, j, k, l)$ represent the cell health. It is basically a measure of how many nutrients it has achieved in its lifetime and of the success quotient towards avoiding obnoxious substances. The bacteria are then sorted according to chemotactic parameters $\mathrm{C}(\mathrm{i})$ with increasing $\operatorname{cost} Z_{\text {health }}$ i.e. lower health. The cell with maximum $Z_{\text {health }}$ values dies while the bacteria with the optimal values are splitted.

Step 7: If $\mathrm{k}<\mathrm{S}_{\mathrm{r}}$, move to step 2 with the number of specified reproduction steps not being reached. Hence, the next generation of the proceeding chemotactic process is initiated.

\section{Step 8: Elimination-Dispersal}

Each cell is eliminated and dispersed with probability Pe for $i=1,2 \ldots, P$ which keeps the total population swarm size constant. To achieve this, if a bacterium is eliminated, one is dispersed into a random location in the search space.

Step 9: If $1<$ Se, move to step 1 , else quit.

\subsection{Particle Swarm Optimization}

Particle Swarm Optimization is a technique used to solve nonlinear problems, developed by
American Social Psychologist James Kennedy. The technique is based on the concept of interaction and behavior among birds or fishes where birds and fishes gather, organize, compete and co-ordinate among themselves to find food $[1,4]$. A similar concept is seen in Particle Swarm Optimization where there is a population known as swarm which consists of a number of random solutions known as particles [15]. These particles are free to move around in the search-space based on certain formulae in a multi-dimensional space based on their previous movements and also considering the movements of their neighbors $[29,27]$.

The particles keep on changing their positions and velocities based on the following formula:

Let $x_{i}=\left[x_{i 1}, x_{i 2}, x_{i 3} \ldots \ldots \ldots \ldots x_{i d}\right]^{T}$ represent the position of the $i^{t h}$ element in the dimension space,

Let $x_{i p b e s t}=\left[x_{i, 1 \text { pbest }}, x_{i, 2 p b e s t}, x_{i, 3 p b e s t} \ldots \ldots . . . x_{i, d p b e s t}\right]^{T}$ represent the previous best position of the $i^{\text {th }}$ element in the 'd' dimension space.

Among the group the best particle of the index is gbest $_{d}$

The velocity of the particle $i^{\text {th }}$ is presented as $V_{i}=\left[\mathrm{V}_{i 1}, \mathrm{~V}_{i 2}, \mathrm{~V}_{i 3} \ldots \ldots \ldots . . . \mathrm{V}_{i d}\right]^{T}$

Then the updated velocity and the distance from pbest $_{\text {id }}$ and gbest $_{d}$ is defined as;

$V_{i d}{ }^{t+1}=w * V_{i d}{ }^{t}+c_{1} * r_{1}^{t}\left(\right.$ pbest $\left._{i d}{ }^{t}-x_{i d}{ }^{t}\right)+c_{2} * r_{2}{ }^{t}\left(\right.$ gbest $\left._{d}{ }^{t}-x_{i d}{ }^{t}\right)$

$x_{i d}{ }^{t+1}=x_{i d}{ }^{t}+V_{i d}{ }^{t+1}$

where $i=1,2,3, \ldots ., m$ and $d=1,2,3, \ldots . ., n$

In the above equation, 'm' represents no. of particles, 'n' represents dimension index, 't' stands for number of iteration, $V_{i d}{ }^{t}$ represents speed of the particles ' $\mathrm{i}$ ' at its $\mathrm{t}$ times in d dimension space. $x_{i d}{ }^{t}$ represents position of the particles ' $i$ ' at its $t$ times in $\mathrm{d}$ dimension space, $x_{i d}{ }^{t+1} \mathrm{In}$ dimension $\mathrm{d}$ of the $i^{\text {th }}$ particle position in iteration $\mathrm{t}+1$, ' $\mathrm{w}$ ' represents the inertia of weight factor, ${ }^{c_{1}}$ and ${ }^{c_{2}}$ represent acceleration constants, ${ }^{r_{1}}$ and ${ }^{r_{2}}$ are random variables $[0,1]$, pbest ${ }_{i d}{ }^{t}$ represents the most optimal position at its ' $t$ ' times in the dimension quantity of the 
individual ' $\mathrm{i}$ ', gbest $_{d}{ }_{d}$ represents the $\mathrm{d}$ dimension quantity of the swarm at its most optimal position.

\subsubsection{PSO Algorithmic Approach for the Specified Design}

For the PZA based system, the filter tuning parameters and PID controller design problem are casted in PSO framework. In the three dimensional search space, $K_{P}, K_{I}$ and $K_{D}$ are considered the three dimensions and the fitness function is based on time domain characteristics, as stated earlier. The number of adaptation varies from 100 to 1000 keeping the population swarm size constant at 50 , acceleration constant at 1.5 and inertia weight factor as 0.7 .

Table 3.PSO based optimization results

\begin{tabular}{|c|c|c|c|c|}
\hline Iteration & \multicolumn{3}{|c|}{$\begin{array}{c}\text { Optimal Best Gain } \\
\text { Points }\end{array}$} & $\begin{array}{c}\text { Optimal } \\
\text { Fitness } \\
\text { Function Value }\end{array}$ \\
\hline 100 & 213.6 & 649.5 & 701.7 & 2.2642 \\
\hline 200 & 621.3 & 1430.7 & 1495.3 & 1.3279 \\
\hline 300 & 1903.7 & 2421.5 & 2425.1 & 1.0105 \\
\hline 400 & 1941.0 & 723.6 & 3338.0 & 0.8708 \\
\hline 500 & 1568.3 & 4222.6 & 4192.8 & 0.7953 \\
\hline 600 & 2125.8 & 4834.6 & 4944.7 & 0.7504 \\
\hline 700 & 699.0 & 5519.8 & 5755.1 & 0.7151 \\
\hline 800 & 5571.4 & 1121.0 & 6472.2 & 0.6912 \\
\hline 900 & 1747.7 & 1056.2 & 7369.2 & 0.6674 \\
\hline 1000 & 4511.0 & 2037.7 & 8014.7 & 0.6544 \\
\hline
\end{tabular}

The value of the points which minimizes the fitness function along with the optimal value of the fitness function using PSO is mentioned in each row of Table 3. It can be observed that the value of function is optimized as the iterations are increased, leading to a value of 0.6544 from 2.2642. The corresponding proportional gain of 2037.7 is then used for determining the filter time constant which turns out to be 2.78 .

From the comparative study of the performance of both swarm intelligence techniques, it can be noticed that the optimization of PSO algorithm is better than that of BFO in the sense that the optimal value of the fitness function for BFO is 8.1652 while PSO provides a much low value of 0.6544 .

\section{Results and Discussion}

The dynamic model of piezoelectric plant has been expressed in the form of a second order mass-spring-damper system with nonlinear hysteresis force, as seen in [20,19]. This work uses a similar mathematical model along with a Dahl based hysteresis model [26], represented by the following equation,

$$
M \dot{x}+D \dot{x}+K x=T u-F_{h}
$$

where $\mathrm{M}$ stands for the system, $\mathrm{D}$ is damping co-efficient, $\mathrm{T}$ is piezoelectric coefficient, $\mathrm{K}$ is stiffness, $\mathrm{u}$ is input voltage and $F_{h}$ represents the hysteresis effect in terms of force. The schematic representation of the PZA system along with Dahl hysteresis model is shown in Figure 5.

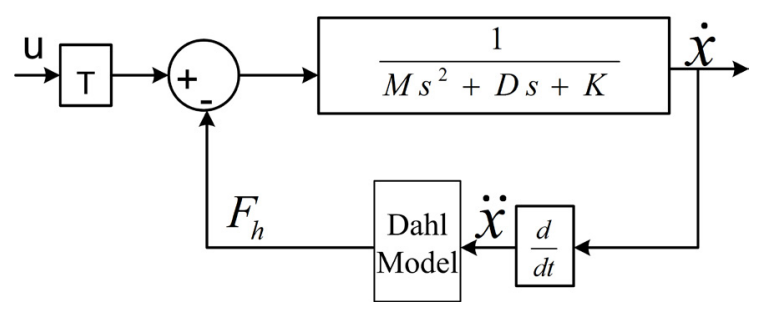

Figure 5. Second order piezo electric plant using

Dahl Model for nonlinear Hysteretic force $F_{h}$.

A preloaded piezo actuator with integrated feedback strain gauge sensor from Physik Instrument has been used in this study. Based on the above system modelling, the transfer function of the actuator has been obtained through model identification with the help of several experimental data with the test bench shown in Figure 6, and is

$$
T F_{\text {Piezo }}=\frac{0.0336}{0.1828 s^{2}+190.154 s+119206.85}
$$

The following section elaborates upon the control performance of the second order PZA system which is observed when it is subject to several real-time constraints.

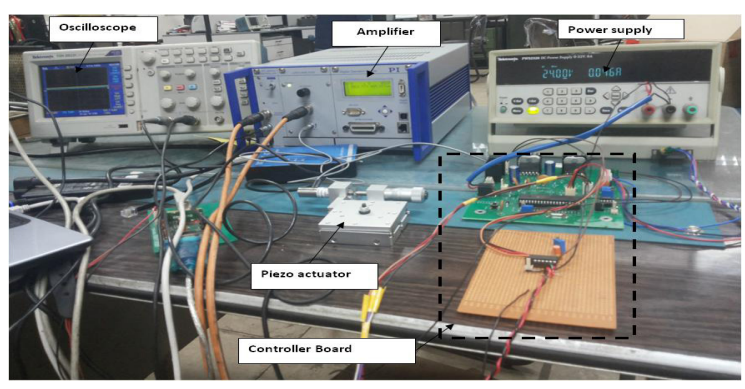

Figure 6. Experimental setup of the PZA 


\subsection{Impulse Disturbance at the PZA System Output}

Initially, the PZA process and model are assumed to be a perfect match, with the system being affected with no disturbance or delay except an unit impulse, as shown in Figure 7. The proposed PSO based M-IMC control regime has bene compared with two of the most widely used classical control genres used for piezoelectric actuation - Classical PID [22, 26] and Classical IMC $[7,16]$ and the system response to each of them is illustrated in Figure 7. The simulation time has been set relatively large in order to observe the complete output of the zero tracking input. The response is seen to track the zero input till the 30th second where the unity impulse disturbance is applied and the response level shifts to one. The controller steadily eliminates this effect and the response gradually returns to zero. Withdrawal of the disturbance at the 60th second makes the system behave similarly in a opposite direction and the outputs again gets to zero owing to controller effect. Classical PID is seen to have the largest settling time of about 51.5 seconds as a response to the disturbance. Classical IMC offers a better response with a settling time of 46.5 while PSO based M-IMC has the least value of 44 seconds.

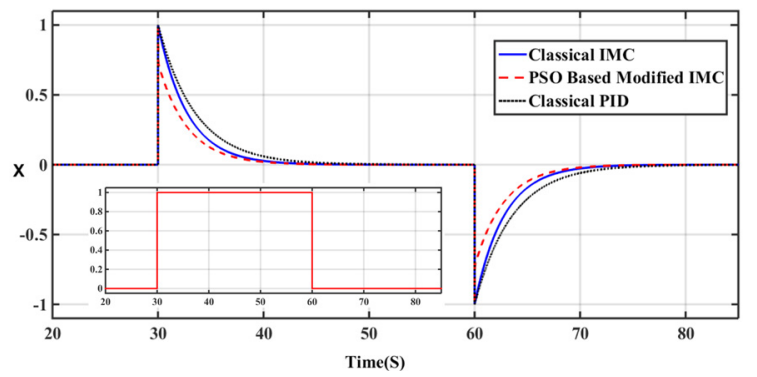

Figure 7. Set point tracking response with impulse disturbances for PZA system.

\subsection{Unit Step Disturbance at the PZA System Output}

An unit step disturbance input at $\mathrm{t}=10 \mathrm{sec}$ is applied to the PZA system to order to observe the performance of the above three control regimes (Figure 8). PSO based Modified Internal Model Control has a settling time of 26 seconds as compared to Classical Internal Model Control of 27.5 seconds. Classical PID is seen to be the least efficient with the largest settling value of 31 seconds.

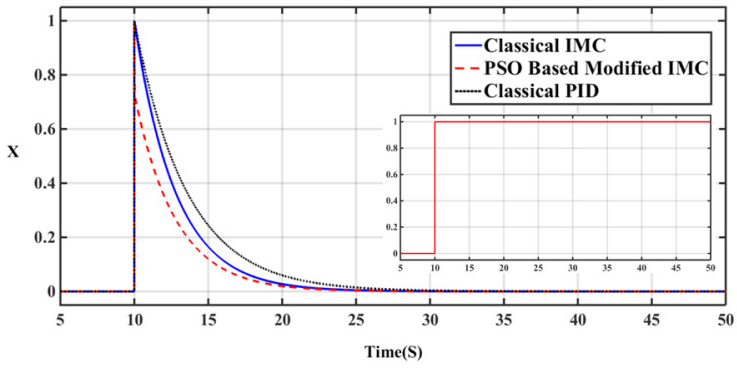

Figure 8. System tracking response with unit step disturbances for PZA system.

\subsection{Gaussian Noise Disturbance at the PZA Plant Output}

Simple transfer model of disturbances is not enough to stand for the random disturbances that effect a plant in real life scenario. White Gaussian noise (mean $=0$, variance $=1$ ) has been used to represent the stochastic disturbance model which is similar to a sinusoid of variable amplitude and frequency. The system output is seen for a zero tracking input in Figure 9 where the controller is seen to mitigate the random effect and oscillates about zero without any instability. PSO tuned M-IMC is seen to quickly nullify the Gaussian noise effect with a fast response time as compared to Classical IMC.

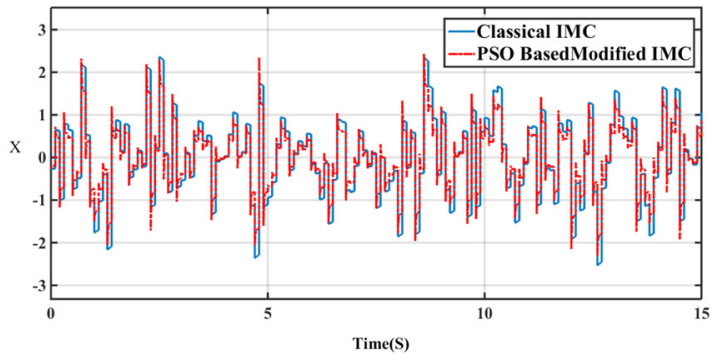

Figure 9. Response of classical IMC and PSO based M-IMC PZA system to Gaussian Disturbance.

\subsection{Plant Parameter Variation at the PZA Plant Output}

As elaborated earlier, plant/model mismatch occurs commonly primarily as system modelling due to the resulting approximations when the physical system is transformed into its mathematical equivalent. Model mismatch is difficult to avoid and occurs for reasons such as inaccurate system identification or inappropriate order assumption of 
the plant. The nonlinear nature of the PZA also enhances possibilities of mismatch.

The following section considers a plant model mismatch of $35 \%$ in plant parameters as compared to the nominal model which has been fed with a unit step input along with an impulse disturbance. The step response of the classical IMC and PSO tuned IMC are seen in Figure 10. The results suggest that PSO based Modified IMC is able to consider the effects of plant parameter variation in a much befitting manner with reduced settling time and a better regulation of its output to track in comparison to classical IMC which is seen to have a steady state value not equal to unity, but slightly greater than it, as it is shown in inset of Figure 10.

Set point tracking at unity of the above controllers with $35 \%$ plant parameter variation and an unit impulse disturbance can also be observed. The PSO based Modified IMC has a shorter settling time of 74 seconds as compared to 78 seconds of classical IMC.

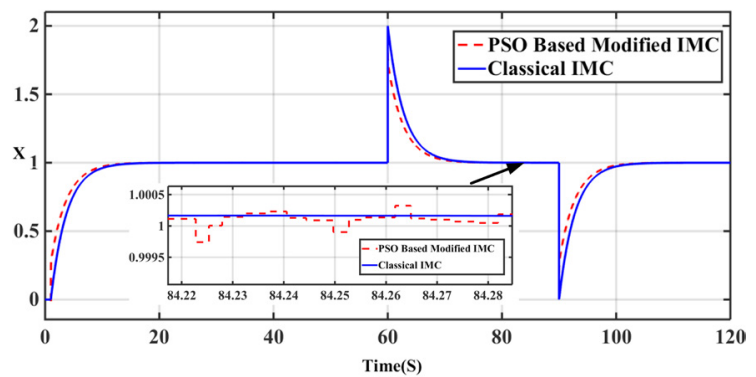

Figure 10. Classical and PSO based M-IMC with $35 \%$ plant parameter mismatch - Step responses

\subsection{System Performance in Presence of Time Delay and Step Disturbance}

A time lag persists between the point of input and actual output in real plant due to its physical charasteristics. The same has been modelled using a delay element in the PZA system whose value is taken to be 1 seconds while it is kept 2 seconds for the plant model.

Fig 11 shows the time response of the classical IMC controller and PSO tuned M-IMC controller where in the former an uneven response is observed and the perturbation effect is seen to be exhibited for a longer time. On the contrary,MIMC offers a much smoother response with a shorter settling time of 18.5 seconds compared to 36.5 seconds.

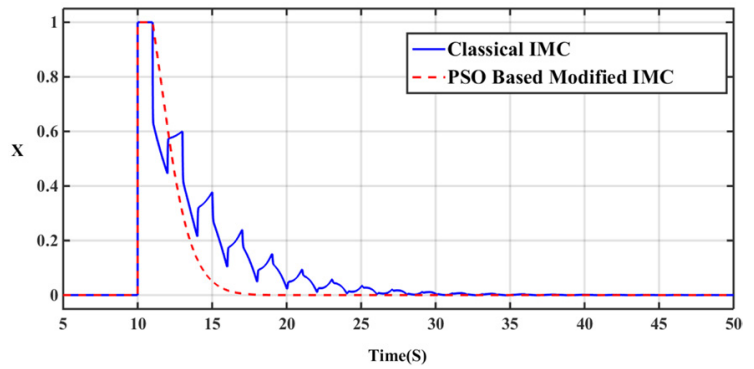

Figure 11. System responses of classical and PSO based M-IMC controller for PZA system with time delay.

\subsection{Step Response of Time Delayed PZA System with Impulse Disturbance}

Performance of classical PID, classical IMC and PSO based Modified IMC towards step response of the piezo plant incorporating a delay of one seconds along with impulse disturbance, illustrated in Figure 12. Classical PID controller has a settling time of 84 seconds which is the largest. PSO based Modified IMC gives a better performance than classical IMC with a settling time of 74.5 seconds and 76.5 seconds respectively.

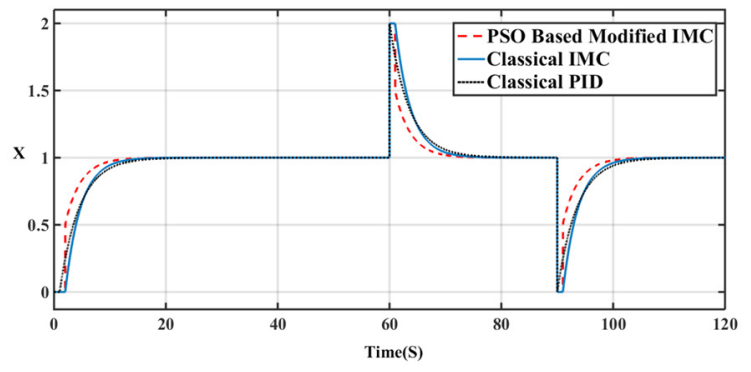

Figure 12. System step response of classical IMC, PID and PSO based M-IMC with impulse disturbances for PZA system.

For an unperturbed PZA system without any time delay, four standard control parameters for a unit step input - Rise time, settling time, Integral of absolute error, $I_{I A E}$ and Integral of Squared Error, $I_{I S E}$ are evaluated to verify the efficiency of PSO based Modified IMC with classical controllers, during $0-50^{\text {th }}$ seconds as seen in Figure 12.

$I_{\text {IAE }}=\int_{0}^{T}|e(t)| d t I_{I S E}=\int_{0}^{T}[e(t)]^{2} d t$

From Table 4, it can be observed that Classical IMC considerably improves the system response 
with the lower time values as well as with the error indices as compared to Classical PID. However, PSO based Modified IMC is seen to have the best performance with a $\mathrm{I}_{\mathrm{ISE}}$ of 314.36 and a settling time of 16.5 seconds.

Table 4. System Performance Parameters of PZA Input against Step Input

\begin{tabular}{|l|l|l|l|l|}
\hline $\begin{array}{l}\text { Control } \\
\text { Schemes }\end{array}$ & $\begin{array}{l}\text { Rise } \\
\text { time } \\
\text { (s) }\end{array}$ & $\begin{array}{l}\text { Settling } \\
\text { time (s) }\end{array}$ & $I_{\text {IAE }}$ & $I_{\text {ISE }}$ \\
\hline $\begin{array}{l}\text { Classical } \\
\text { PID }\end{array}$ & 7.62 & 23.5 & 1108.7 & 679.56 \\
\hline $\begin{array}{l}\text { Classical } \\
\text { IMC }\end{array}$ & 6.09 & 19 & 830.38 & 483.36 \\
\hline $\begin{array}{l}\text { PSO Based } \\
\text { Modified } \\
\text { IMC }\end{array}$ & 5.495 & 16.5 & 637.16 & 314.36 \\
\hline
\end{tabular}

\section{Conclusion}

Bio-inspired evolutionary computational techniques are being used in several industrial and engineering applications with remarkable results. This paper explores the performance of a Modified Internal Model Control on the control response of a piezo electric actuator which is seen to be

\section{REFERENCES}

1. Bai, Q. (2010). Analysis of Particle Swarm Optimization Algorithm, Computer and Information Science, 3(1).

2. Bashash, S. \& Jalili, N. (2009). Robust adaptive control of coupled parallel piezoflexural nanopositioningstages, IEEE/ASME Transactions on Mechatronics, 14(1), 11-20.

3. Colgate, J. E. \& Hogan, N. (1988). Robust control of dynamically interacting systems, International Journal of Control, 48(1), 65-88.

4. Clerc, M. \& Kennedy, J. (2002). The particle swarm-explosion stability and convergence in a multidimensional complex space, IEEE Transactions on Evolutionary Computation.

5. Dasgupta, S., Das, S., Abraham, A. \& Biswas, A. (2009). Adaptive Computational Chemotaxis in Bacterial Foraging Optimization, IEEE Transactions on Evolutionary Computation, 13(4). better than classical Internal Model Control. Two bio inspired swarm intelligence-based algorithm are then used to optimise a fitness function which is utilized to determine the Modified Internal Model Control parameters, including filter time constant. Particle Swarm Optimization provides promising results as compared to Bacterial Foraging Optimization. Hence, it is used to tune the M-IMC controller, termed as PSO based M-IMC. From the results, it is evident that the proposed controller is able to track the desired set point accurately. The plant performance has also been observed against several disturbances. Results of PSO based M-IMC have a reduced settling time, lower overshoot and an overall better response to the external disturbances compared to other controllers.

\section{Acknowledgements}

The research reported in this paper has been supported by the "Indo French Center for The Promotion of Advanced Research (IFCPAR/CEFIPRA)". Authors also gratefully acknowledge for the continuous support of Director of CSIR-CMERI.

6. Devasia, S., Eleftheriou, E. \& Reza Moheimani, S. O. (2007). A Survey of Control Issues in Nanopositioning, IEEE Transactions on Control Systems Technology, 15.

7. Huang, Y. C. \& Cheng, C. H. (2003). Internal model control approach for designing piezoelectric actuator controller with nonminimum phase. In Proceedings of the IEEE International Symposium on Intelligent Control, Houston, TX, USA (pp. 188-193).

8. Ketabchi, H. \& Ataie-Ashtiani, B. (2015). Evolutionary algorithms for the optimal management of coastal groundwater, Journal of Hydrology, 520(1), 193-213.

9. Li, D., Liu, L. \& Jin, Q. (2015). Maximum sensitivity based fractional IMC-PID controller design for non-integer order system with time delay, Journal of Process Control. 
10. Liu, T. \& Gao, F. (2011). Enhanced IMC design of load disturbance rejection for integrating and unstable processes with slow dynamics, ISA Transactions.

11. Manikandan, P. \& Ramyachitra, D. (2017). Bacterial Foraging OptimizationGenetic Algorithm for Multiple Sequence Alignment with MultiObjectives Nature, Scientific Reports, 7. Article number: 8833 .

12. Morari, M. \& Zafirious, E. (1989). Robust Process Control. Prentice-Hall.

13. Muniraj, R., Willjuice Iruthayarajan, M. \& Arun, R. (2017). Tuning of Robust PID Controller with Filter for SISO System Using Evolutionary Algorithms, Studies in Informatics and Control, 26(3), 277286. ISSN 1220-1766.

14. Nanda, S. J. \& Panda, G. (2014). A survey on nature inspired metaheuristic algorithms for partitional clustering, Swarm Evol. Comput., 16(1).

15. Panigrahi, S. K., Sahu, A. \& Pattnaik, S. (2015). Structure Optimization Using Adaptive Particle Swarm Optimization, Procedia Computer Science, 48, 802-808.

16. Rakotondrabe, M., Agnus, J. \& Lutz, P. (2011). Feedforward and IMC-feedback controlofanonlinear2-DOF piezoactuator dedicated to automated micropositioning tasks. In IEEE International Conference on Automation Science and Engineering, Trieste (pp. 393-398). doi: 10.1109/CASE.2011.6042415.

17. Ray, P. K. \& Subudhi, B. (2012). BFO optimized RLS algorithm for harmonics estimation, Appl. Soft Comput., 12(8), 1965-1977.

18. Rupp, D. \& Guzzella, L. (2010). Iterative Tuning of Internal Model Controllers With Application to Air/Fuel Ratio Control, IEEE Transactions on Control Systems Technology.

19. Shome, S. K., Pradhan, S., Mukherjee, A. \& Datta, U. (2013). Dither based precise position control of piezo actuated micro-nano manipulator.
In Proceedings of the $39^{\text {th }}$ Annual Conference of the IEEE Industrial Electronics Society (IECON '13) (pp. 3486-3491).

20. Shome, S. K., Prakash, M., Pradhan, S. \& Mukherjee, A. (2015). On Synergistic Integration of Adaptive Dithering Based Internal Model Control for Hysteresis Compensation in Piezoactuated Nanopositioner, Mathematical Problems in Engineering, vol. 2015, Article ID 365141.

21. Subudhi, B. \& Pradhan, R. (2018). Bacterial Foraging Optimization Approach to Parameter Extraction of a Photovoltaic Module, IEEE Transactions on Sustainable Energy, 9(1).

22. Sung, B., Lee, E. \& Kim, I. (2008). Displacement Control of Piezoelectric Actuator using the PID Controller and System Identification Method. In Joint International Conference on Power System Technology and IEEE Power India Conference, New Delhi (pp. 1-7). doi: 10.1109/ICPST.2008.4745381.

23. Vasiliu, N., Călinoiu, C., Vasiliu, D. \& Ion Guță, D. D. (2007). High Accuracy Electro Hydraulic Servo Mechanisms with Additional Derivative Feedback. In Recent Advances in Aerospace Actuation Systems and Components, June 13-15, 2007, Toulouse (pp.183-188). ISBN 9782-87649-053-6.

24. Vasiliu, N., Vasiliu, D., Călinoiu, C. \& Puhalschi, R. (2018). Simulation of the Fluid Power Systems with Simcenter Amesim. Boca Raton, FL, USA, CRC Press, Taylor \& Francis Group.

25. Verma, S. W.-J., Kim, W. J. \& Shakir, H. (2005). Multi-axis Maglev nano positioner for precision manufacturing and manipulation applications, IEEE Trans. Ind. Appl., 41(5), 1159-1167.

26. Xu, Q. \& Li, Y. (2010) Dahl Model-Based Hysteresis Compensation and Precise Positioning Control of an XY Parallel Micromanipulator With Piezoelectric Actuation, Journal of Dynamic System, Measurement and Control. ASME. 
27. Xu, X., Pan, Z., Xi, Y. \& Chen, L. (2012). Incremental Particle Swarm Optimization, Physics Procedia, 24(Part B).

28. Yi, J., Chang, S. \& Shen,Y. (2009). Disturbance-observer-based hysteresis compensation for piezoelectric actuators, IEEE/ASME Transactions on Mechatronics, 14(4), 456-464.

29. Yoshinda, H., Kawatta, K., Fukuyama, Y., Takayama, S. \& Nakamishi, Y. (2007). A particle swarm optimization for reactive power and voltage control considering voltage security assessment, IEEE Transactions On Power systems.

30. Zou, Q. K. K., Leang, K .K., Sadoun, E., Reed, M. J. \& Devasia, S. (2004). Control issues in high-speed AFM for biological applications: Collagen imaging example, Asian J. Control: Special Issue Adv. Nano-Technol. Control, 6(2), 164-178. 